atic infants or observe for signs of sepsis before initiating treatment. One could treat all infants with risk factors at birth or not treat any until they became symptomatic. Most approaches fall somewhere in between. There is a tradeoff between treating infants with antibiotics who do not have infection and delaying treatment in those who do. Rational decisions require a value judgment based on the perceived utility/harm of these 2 outcomes as well as the probability of infection. An advantage to our approach is that the EOS calculator provides an explicit estimate of infection risk for each infant to help guide such decisions. Other approaches, such as those from the Centers for Disease Control and Prevention (CDC) and American Academy of Pediatrics, do not. ${ }^{1-3}$ While we have set thresholds for a blood culture at a risk of infection of 1 in 1000 and for antibiotics at 3 in 1000, these thresholds can be set differently if clinicians choose to do so.

When looking at utility/harm, we need to consider the entire population, not just the infants with EOS. Using the EOS calculator, we reduced antibiotic usage by $2.4 \%$, meaning 1350 fewer infants received empirical antibiotics compared with our prior approach based on CDC guidelines. ${ }^{4}$ In contrast, treatment was delayed in 2 infants (0.004\%) compared with CDC guidelines. Neither of these infants had complications, and repeated cultures in both were sterile before antibiotics were initiated. Similarly, applying our antibiotic use rate to the baseline period, 2293 fewer infants would have received antibiotics, while 3 infected infants would have had their antibiotics delayed until they became symptomatic. Thus, for every infant with EOS in whom antibiotics are delayed compared with CDC guidelines, there are approximately 700 in whom unnecessary antibiotics could be avoided.

Infants whose examination was equivocal at birth had abnormalities, such as tachypnea, not requiring respiratory support and did not meet our criteria for clinical illness. If they had, their risk estimates would have placed them above our antibiotic treatment threshold. The utility of starting antibiotics at birth in asymptomatic infants is unclear. In the EOS calculator period, severe complications occurred only among infants who were ill at birth.

While additional data on outcomes, including sequelae of early antibiotic exposure, will be useful, we believe explicit estimates of sepsis risk and numbers needed to treat should guide decisions on when to begin antibiotics.

Michael W. Kuzniewicz, MD, MPH

Gabriel J. Escobar, MD

Karen M. Puopolo, MD, PhD

Author Affiliations: Perinatal Research Unit, Division of Research, Kaiser Permanente Northern California, Oakland (Kuzniewicz, Escobar); Department of Pediatrics, University of California, San Francisco (Kuzniewicz); The Permanente Medical Group, Oakland, California (Kuzniewicz, Escobar); Systems Research Initiative, Division of Research, Kaiser Permanente Northern California, Oakland (Escobar); Newborn Care at Pennsylvania Hospital, The Children's Hospital of Philadelphia, Philadelphia, Pennsylvania (Puopolo); Perelman School of Medicine, University of Pennsylvania, Philadelphia (Puopolo).

Corresponding Author: Michael W. Kuzniewicz, MD, MPH, Perinatal Research Unit, Division of Research, Kaiser Permanente Northern California, 2000 Broadway Ave, Oakland, CA 94612 (michael.w.kuzniewicz@kp.org).
Published Online: August 14, 2017. doi:10.1001/jamapediatrics.2017.2467 Conflict of Interest Disclosures: None reported.

1. Verani JR, McGee L, Schrag SJ; Division of Bacterial Diseases, National Center for Immunization and Respiratory Diseases, Centers for Disease Control and Prevention (CDC). Prevention of perinatal group B streptococcal disease: revised guidelines from CDC, 2010. MMWR Recomm Rep. 2010;59(RR-10): $1-36$

2. Polin RA; Committee on Fetus and Newborn. Management of neonates with suspected or proven early-onset bacterial sepsis. Pediatrics. 2012;129(5): 1006-1015.

3. Brady MT, Polin RA. Prevention and management of infants with suspected or proven neonatal sepsis. Pediatrics. 2013;132(1):166-168.

4. Kuzniewicz MW, Puopolo KM, Fischer A, et al. A quantitative, risk-based approach to the management of neonatal early-onset sepsis. JAMA Pediatr 2017;171(4):365-371.

\section{The UK Royal College of Paediatrics and Child Health's Policy on Funding From Commercial Organizations}

To the Editor We read with interest the Viewpoint on relationships between the American Academy of Pediatrics and infant formula companies. ${ }^{1}$ The United Kingdom has a very different health system from the United States and prohibits formula gift bags and advertising of breast milk substitutes. In 2016, we undertook a consultation with Royal College of Paediatrics and Child Health (RCPCH) members about acceptance of funding from commercial organizations, leading to revised processes that may be of interest to pediatricians globally.

We encourage organizations, including infant formula companies, to support RCPCH activities benefiting infants, children, and young people. We will conduct a formal due diligence process, defined by the UK Charity Commission as "the range of practical steps that need to be taken by trustees so that they are reasonably assured of the provenance of the funds given to the charity; confident that they know the people and organizations the charity works with; and able to identify and manage associated risks." ${ }^{2}$ We require donors (1) meet or demonstrate commitment to meeting relevant Codes of Practice (eg, The Association of the British Pharmaceutical Industry Code of Practice for the Pharmaceutical Industry and the World Health Organization International Code of Marketing of BreastMilk Substitutes); (2) show willingness to share information on breaches in the United Kingdom and, internationally, of relevant code(s), criminal investigations or prosecutions, and remediating actions; (3) provide a reasonable explanation should inconsistencies in information be identified; (4) provide evidence of robust governance of national businesses by the parent company; and (5) sign off responses by an internationallevel senior director. Donations will be open and acknowledged, but RCPCH will not permit the involvement of companies or linking of names or logos to specific outputs. We will only allow advertising of specialist formula, not products for healthy full-term infants. A subgroup of the RCPCH independent trustee board scrutinizes due diligence responses. The first 4 infant formula companies considered failed to provide necessary assurances and will not be permitted to exhibit at RCPCH events, including our annual conference, or contribute to $\mathrm{RCPCH}$ funds until such time as they are able to provide these 
assurances. Details are available on the RCPCH website (http: //www.rcpch.ac.uk/).

The RCPCH rejects the promotion of infant formula and is proud to be tackling serious obstacles to breastfeeding. We advocate for national employment laws and societal attitudes that support women to breastfeed without detriment to their careers or their dignity; health education at schools; and national campaigns directed at parents and the public. We accept a woman's right to choose how she feeds her infant and the necessity for high-quality products for healthy babies as well as those with special needs. We want interactions with industry to be transparent so that professional advice is unbiased and practice is not influenced by pressure groups. We encourage open involvement of pediatricians in collaborative research to improve products and the evidence base for infant nutrition. We hold industry to the highest standards and believe isolationism and refusal to engage makes it easier for bad practices to be perpetuated. Dialogue and transparent collaboration is the way to benefit babies around the world.

\section{Neena Modi, MBChB, MD, FRCP, FRCPCH, FRCPE Judith Ellis, MBE, PhD, MSc, RN Mary Marsh, MBE, BSc}

Author Affiliations: Imperial College London, London, England (Modi); Royal College of Paediatrics and Child Health, London, England (Modi, Ellis, Marsh).

Corresponding Author: Neena Modi, MBChB, MD, FRCP, FRCPCH, FRCPE, Royal College of Paediatrics and Child Health, 5-11 Theobald's Rd, London WC1X 8SH, United Kingdom (president@rcpch.ac.uk).

Published Online: August 21, 2017. doi:10.1001/jamapediatrics.2017.2470 Conflict of Interest Disclosures: Dr Modi reports awards outside the submitted work from the Medical Research Council, National Institute of Health Research, British Heart Foundation, Westminster Medical School Research Trust, HCA International, Nestlé Research, Bliss, NHS London, NHS Improvement, NHS Litigation Authority, Healthcare Quality Improvement Partnership, and Department of Health; personal fees from Alexion; and conference travel and accommodation from Prolacta. Dr Modi has a patent relating to Infant Body Composition pending and her employer, Imperial College London, has established a research and innovation partnership with Nestlé Research. Dr Ellis is chair of Tropical Health Education Trust that receives funding from commercial organizations. No other disclosures are reported.

Editorial Note: This letter was shown to the corresponding author or the original article, who declined to reply on behalf of the authors.

1. Sharfstein JM, Silver DL. Relationship between the American Academy of Pediatrics and infant formula companies. JAMA Pediatr. 2017;171(7): 613-614.

2. Charity Commission for England and Wales. Compliance Toolkit: Protecting Charities from harm. Due diligence, monitoring and verifying the end use of charitable funds. https://www.gov.uk/government/uploads/system/uploads /attachment_data/file/550682/Chapter_2_Summary.pdf. Published 2016. Accessed July 24, 2017.

\section{Neurodevelopmental Outcome in Relation to Treatment of Patent Ductus Arteriosus}

To the Editor We read with great interest the study by Weisz et $\mathrm{al}^{1}$ that evaluated the association of patent ductus arteriosus (PDA) ligation with death or neurodevelopmental impairment in preterm infants born prior to 28 weeks' gestation. ${ }^{1}$ The authors were correctly aware that their estimate of the effect of PDA ligation on mortality (adjusted odds ratio, 0.09; 95\% CI, 0.04-0.21) was likely biased by survivor treatment selection (immortal time bias). ${ }^{2}$ Immortal time bias results from misclassification of the follow-up period when the outcome (mortality) cannot occur in exposed (ligated) patients, who must by default survive until ligation. We are concerned the method used to account for it, a subcohort analysis, was undersensitive. As we have shown, day-specific mortality in infants at risk for PDA decreases rapidly in the first postnatal weeks. ${ }^{3}$ Given the authors' uniquely rich data set and ability to control for confounding by indication, we suggest applying timedependent Cox regression, a more advanced and validated method $^{4}$ that considers treatment as a time-dependent exposure. Using a postnatal day time-scale, infants with hemodynamically significant PDA would enter the life table as treated on the day they received their first nonsteroidal anti-inflammatory drug (NSAID) dose. They would continue accruing person-time as medically treated until they died, were discharged, were lost to follow-up, or received surgical ligation. The day that an infant received ligation, they would be withdrawn from the NSAID group and begin to accrue person-time as a ligated patient until death, discharge, or loss to follow-up. Infants ligated without prior NSAID treatment would only accrue person-time in the ligated group, beginning on the ligation day.

The authors' ability to incorporate each patient's daily respiratory support data are another data set strength. However, each patient's probability of receiving NSAID and/or surgical ligation is dependent on their daily respiratory status, which may vary over time and be partially attributable to prior treatment. Marginal structural models incorporate time-varying treatment exposures by weighting the stabilized inverse probability of treatment. ${ }^{5}$ Marginal structural models would allow adjustment for time-dependent risk factors, including daily mean airway pressure and respiratory modality, which predict subsequent treatment. In addition, marginal structural models would account for the effects of past NSAID treatment on subsequent (1) respiratory support and (2) risk of further NSAID or surgical treatment. All described procedures are available in standard statistical packages.

Although randomized trials may be indicated to evaluate PDA ligation effectiveness, rigorous observational analyses are a critical first step. We commend the authors for their important study.

Jonathan L. Slaughter, MD, MPH

Mark A. Klebanoff, MD, MPH

Author Affiliations: Nationwide Children's Hospital, The Ohio State University, Columbus.

Corresponding Author: Jonathan L. Slaughter, MD, MPH, Research 3 Bldg, Nationwide Children's Hospital, 575 Children's Crossroad, Columbus, OH 43215 (jonathan.slaughter@nationwidechildrens.org).

Published Online: August 28, 2017. doi:10.1001/jamapediatrics.2017.2597

Conflict of Interest Disclosures: None reported.

1. Weisz $D E$, Mirea $L$, Rosenberg $E$, et al. Association of patent ductus arteriosus ligation with death or neurodevelopmental impairment among extremely preterm infants. JAMA Pediatr. 2017;171(5):443-449. 